\title{
Uso de la Fauna al Momento del Contacto Hispano-Indígena en el Río de La Plata: un Aporte Arqueozoológico y Etnohistórico
}

\author{
The Fauna Used in the Hispanic-Indian Contact in the Rio De La Plata: \\ Ethnoarchaeological and Ethnohistorical Approach
}

\section{Laura Beovide ${ }^{\mathrm{i}}$ F Fabiana Operti ${ }^{\mathrm{i}}$}

\section{RESUMEN}

Se expone el análisis de un conjunto arqueofaunístico del siglo XVI, sitio Puerto La Tuna, margen izquierda del Río de la Plata. Especies terrestres y acuáticas se identifican en dicho conjunto con evidencias de uso humano. Los resultados se comparan con la presencia y uso de la fauna mencionada en las crónicas históricas para los grupos indígenas vinculados al área. La conjunción de ambas líneas de evidencia (etnohistórica y arqueológica) se presentan como una vía de acercamiento a los distintos aspectos paleoecológicos y de interacción entre los seres humanos y los animales al momento del contacto hispano-indígena.

Palabras Clave:Arqueozoología, Contacto Hispano-Indígena, Arqueología del Río de la Plata.

\begin{abstract}
We present the analysis of the $16^{\text {th }}$ Century archaeofauna from Puerto La Tuna site located on the left bank of the Río de la Plata. Terrestrial and aquatic species were identified together with the evidence of human use. The results were compared with the presence of wildlife and their use by the indigenous groups in the area, both mentioned in the historical chronicles. The combination of these lines of evidence (ethnohistorical and archaeological) is presented as a way to approach the paleoecological aspects and the interaction between humans and animals at the time of the Spanish-Indian contact.
\end{abstract}

Key Word: Archaeozoology, Spanish-Indian Contact, Archeology of the Rio De La Plata.

i Museo Nacional de Antropología, Avda. de las Instrucciones 948, Montevideo, Uruguay. Correo-e: beovide@mec.gub. uy, fabianaoperti@gmail.com.

Recibido: 05-12-2012 Revisado: 29-05-2013 Aceptado: 01-03-2014 


\section{INTRODUCCIÓN}

Las crónicas del siglo XVI mencionan la presencia indígena en el Río de la Plata, pero pocos son los sitios arqueológicos (en su margen izquierda) que pueden asociarse a ese momento histórico (Beovide 2007). Uno de ellos es el sitio Puerto La Tuna $\left(34^{\circ} 42^{\prime} 19.73^{\prime \prime}\right.$; $56^{\circ} 25^{\prime} 04.91^{\prime \prime} \mathrm{W}$, Figura I) con una secuencia de ocupación basada en fechados radiocarbonicos entre ca. 5.000 a 400 años AP (Beovide 20l lb). La excavación VII ubicada en el área más tardía de ocupación del sitio presenta un contexto con arqueofauna y materiales arqueológicos no europeos asociados a un fechado por ${ }^{14} \mathrm{C}$ sobre muestras de carbón en $1.540 \pm 65 \mathrm{cal}$ d.C. (AA88-79I) (Beovide 20I Ib; Malán et al. 20II). Se presenta el análisis del conjunto arqueofaunístico complementado con el análisis etnohistórico (vinculado al proceso carnicero) con el fin de acceder al modo en que las sociedades prehispánicas se vincularon con la fauna.

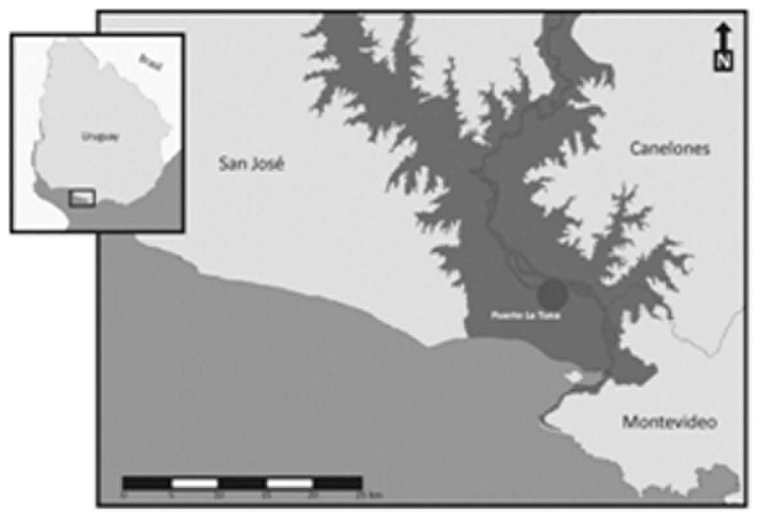

(a)

\section{METODOLOGÍA}

La muestra comprende el total de la arqueofauna (NR) de la excavación VII $(4 \chi 4 \times 0,70$ $\mathrm{m})$ del sitio Puerto La Tuna. El análisis abarcó: a) identificación taxonómica, hábitat, estructura de los recursos y disponibilidad potencial b) estudio espacial y medidas de cuantificación y c) identificación de las modificaciones antrópicas y naturales.

La identificación taxonómica se realizó en base a la colección comparativa del Museo Nacional de Antropología (Uruguay). La estructura de los recursos (Butzer 1989) se analizó en función de los antecedentes paleoecológicos vinculados al área, tomando en cuenta las fuentes históricas (siglos XVI$\mathrm{XVII}$ ) en relación a la fauna descrita por los cronistas y el uso indígena de la misma.

Se tomó en cuenta la distribución y contexto de los restos en la planta de excavación por nivel y por especie. La abundancia taxonómica y anatómica se cuantificó considerando NISP, NMI y MNE (Binford 1980, Mengoni 1988, Payne 1975). El cálculo del MNE se efectuó mediante el registro de zonas diagnósticas (Binford 1984, Mengoni 1999).

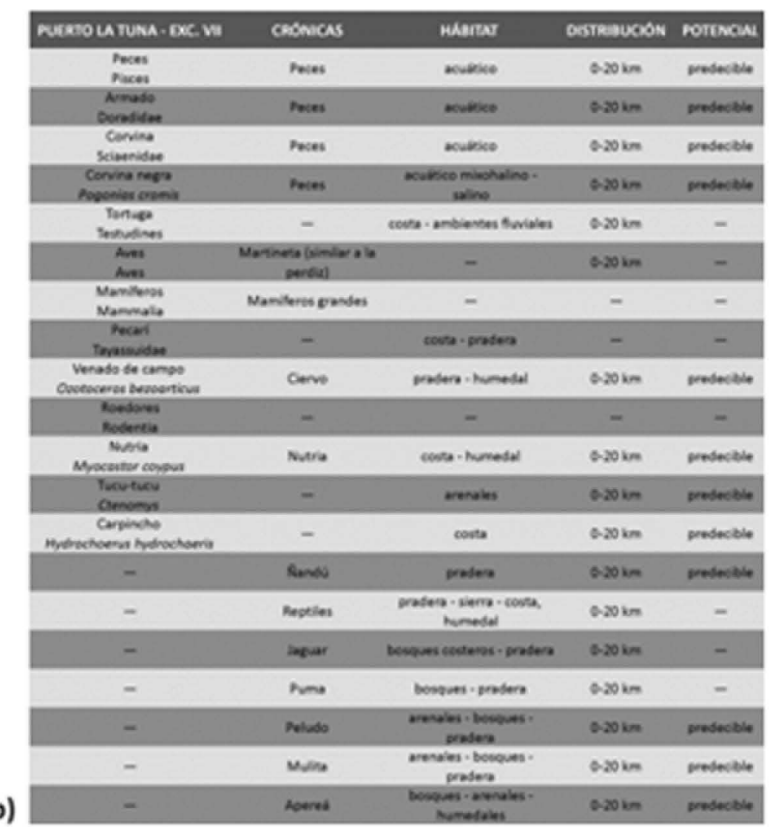

Figura 1: (a) Ubicación del Sitio Puerto La Tuna. (b) Arqueofauna y Fauna referida en las crónicas históricas. Figure 1: (a) Puerto La Tuna Site location (b) Arqueofauna and Fauna mentioned in the historical chronicles. 
Se consideraron las modificaciones antrópicas como marcas de carnicería, tipos de fractura (Binford 198I) y alteración térmica (De Nigris 2004), además de alteraciones naturales como huellas de carnívoros (Binford I98I, Mondini 2002), roedores y raíces (Binford I98I), observadas a simple vista y mediante lupa binocular estereoscópica (BI0058) a 30X y 60X. Se consideraron además aspectos vinculados con la matriz sedimentaria $(\mathrm{pH})$. La meteorización (Behrensmeyer 1978) se valoró como presencia/ausencia por taxón identificado por las características de la muestra (taxones menores a $5 \mathrm{~kg})$.

\section{RESULTADOS}

La muestra se compone de NR: 996 (NR: número total de restos de la muestra con y sin asignación taxonomica), de los cuales 77 I (77,4 \%) son fragmentos óseos de tamaño menor o igual a $1 \mathrm{~cm}$ de largo máximo (100\%) con alteración térmica $(726=94 \%)$ y $\sin$ asignación posible de taxón. Los 225 (22\%) especímenes identificados (NISP) corresponden a 12 taxones. Se identifican cinco especies: Poogonias cromis (corvina negra), Ozotoceros bezoarticus (venado de campo), Myocastor coypus ("nutria" o coipo), Hydrocoerus hydrochaeris (carpincho), Ctenomys sp. (tucu-tucu); dos familias: Doradidae (armado) y Tayassuidae (pecari); dos órdenes: Rodentia (roedor) y Testudines (tortuga) y tres grupos de vertebrados: Peces, Mamíferos y Aves (figura 2).

(a)
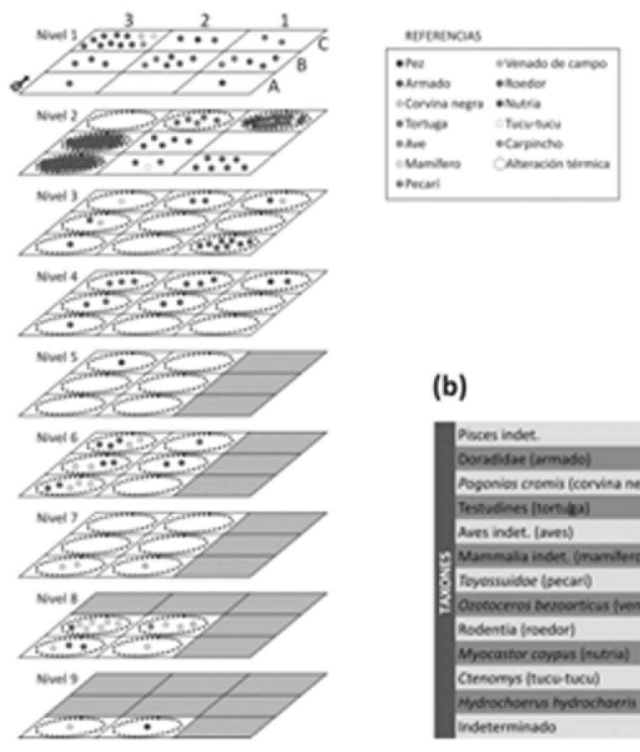

(b)

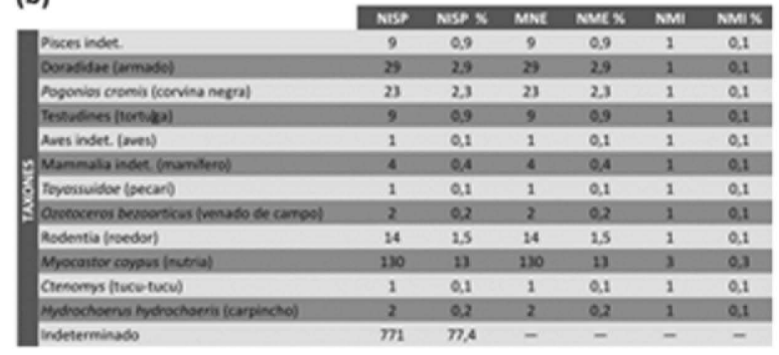

Figura 2: (a) Distribución de la arqueofauna (excavación VII). (b) Medidas de cuantificación por taxón.

Figure 2: (a) Archaeofauna distribution (excavation VII). (b) Measures of quantification by taxon.

Las unidades anatómicas por taxones corresponden a: dientes de corvina negra, astrágalos derecho e izquierdo de venado de campo, maxilar y dientes de coipo, bula timpánica de carpincho, diente de tucu-tucu, placas óseas de armado, malar de pecarí, falanges de roedores, placas dérmicas de tortuga, vértebras de peces, fémur de ave y huesos largos de pequeños mamíferos. Estas se distribuyen en toda la profundidad de la excavación. Sin embargo, los restos de venado de campo se recuperan en los niveles más profundos (7 y 8). La abundancia anatómica y taxonómica del conjunto se detallan en la figura $2 b$.

Las especies recuperadas son endémicas de ecosistemas de pradera, monte ribereño, humedales 
mixohalinos y arenales costeros y son pasibles de localizarse en un ciclo anual en un radio de 20 $\mathrm{km}$ desde el sitio. Por ejemplo, la corvina negra se adentra en el río Santa Lucía para reproducirse de octubre a marzo.

La información etnohistórica en relación a la fauna fue comparada con las especies identificadas en el registro arqueológico y se observan similitudes entre ambos tipos de registros (Figura Ib). En cuanto al venado de campo las crónicas hablan de la abundancia de las manadas de venados en las praderas circundantes a mediados del siglo XVI (Lope de Sousa 1958).

En los primeros niveles de la excavación (2 al 4) se identificó la mayor diversidad de taxones $(\mathrm{N}=9)$ y la menor proporción de restos indiferenciados fragmentados con alteración térmica (208-28\%- de un total de 77I).

Las modificaciones naturales con respecto a la totalidad de la muestra comprenden principalmente la acción de las raíces (NR: 176) -figura $3 c-$ en una proporción mayor en los primeros niveles ( 2 al 4) de la excavación (142, $80 \%$ ). Sólo se reconocen marcas de dientes de carnívoros -furrows- (Binford 1981:48) en un astrágalo derecho de venado de campo (figura 3d). La presencia de meteorización (relevada sólo en especímenes no quemados) se identifica en todos los niveles y en el $27 \%$ de la totalidad del conjunto. En la matriz sedimentaria ( $10 \mathrm{~g}: 9,62 \mathrm{~g}$ de arcillas $\mathrm{y}$ arenas y $0,38 \mathrm{~g}$ de materia orgánica) el valor del $\mathrm{pH}$ en sedimentos es 5 , lo que implica un suelo ácido y poco favorable para la preservación del conjunto arqueofaunístico (Hedges y Millard 1995).

Las marcas vinculadas a los procesos de carnicería (figura 3a-b) se presentan en 3 maxilares de coipo, en un maxilar de roedor y en un malar de pecarí (rascados e incisiones: desarticulación); en una bula timpánica de carpincho (incisiones: desarticulación) y en un astrágalo izquierdo de venado de campo (rascados e incisiones: desarticulación y descarnado). A su vez las evidencias de fracturas en el total de la muestra (NR), en relación a los huesos largos (que en este caso no pudieron asociarse con especies) son NR $=54$ (transversales NR $=24$, longitudinales $N R=14$, en espiral $N R=16$ ). Las fracturas en espiral, asociadas a los procesos de carnicería (Binford I98I), se ubican en los niveles medios $(4,5$ y 6$)$.
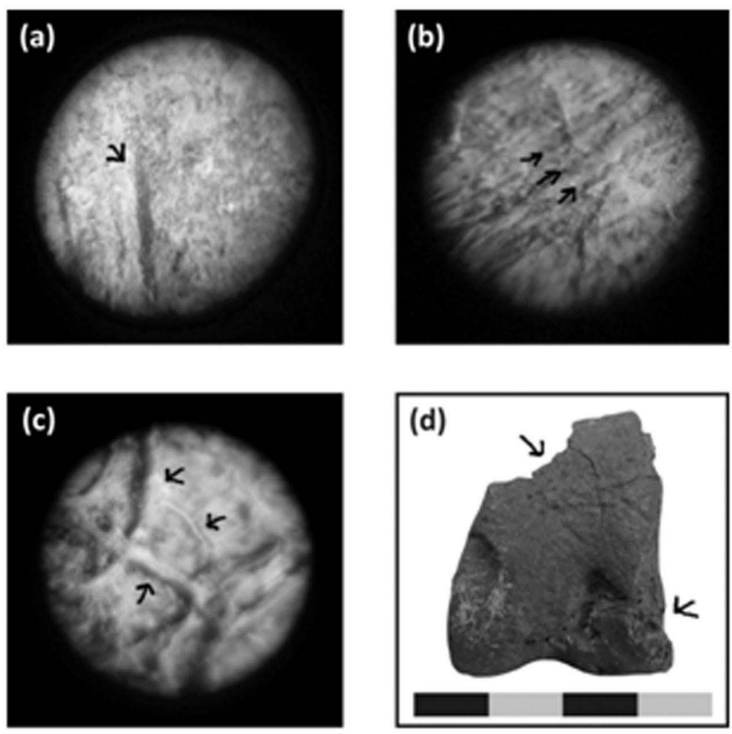

Figura 3: (a-b) Marcas de carnicería observadas con lupa binocular a 30X. (c) Marcas de raíces observadas con lupa binocular a 30X. (d) Marcas de carnívoro.

Figure 3: (a-b) butchery marks observed at 30X binocular. (c) roots marks observed with a $30 \mathrm{X}$ binocular microscope. (d) carnivore marks.

En cuanto a los restos con alteración térmica (NR: $726=73 \%$ ) son más abundantes en los niveles inferiores de la excavación (NR: $518=71 \%$ ). Los colores varían de blanco -Munsell 5YR8/I- (435 = $60 \%)$ a gris oscuro -Munsell 7,5 R 4/0- (34 = 5\%) predominando el primero, lo que puede sugerir un intensa exposición al calor. Sin embargo, la relación color/temperatura/tiempo deberá ser ajustada en función de la particularidad de la muestra.

\section{DISCUSIÓNY CONCLUSIONES}

Del análisis de las crónicas se desprenden rasgos de las economías indígenas del siglo $\mathrm{XVI}$ como presencia de cultivos, posible manejo de la fauna o industrialización de alimentos (Beovide 2003; 2007), con evidencias que se proyectan a más de tres milenios atrás para algunos de éstos (Beovide 20l la). Tomando en cuenta trabajos previos de análisis de las crónicas del siglo $\mathrm{XVI}$ al XVIII en relación a los procesos carniceros de la región (Beovide 2007), y considerando las más relevantes en relación a la ubicación del sitio escritas durante el siglo XVI, como la de Diego de García (1933) entre 1526 y I527, Gonzalo Fernández de Oviedo (I85I) en I535, 
Pero Lope de Souza (1958) entre I530-I532, Ulrich Schmídel (1962) entre I534-I554, se concluye que no se mencionan detalles de cómo se procesaban los grandes animales como el ciervo. Sin embargo, pueden hacerse algunas observaciones acerca de qué animales se cazaban (sobre todo ciervos, nutrias, ñandúes, etc.), con qué finalidad (utilizar sólo la carne o el cuero), con qué instrumentos (boleadoras, arco y flecha) y la forma de caza (a campo abierto, individual o colectivamente) empleada (Beovide 2007). En algunos casos se describe la forma de comer la carne y de aprovechar el cuero para la vestimenta - la habitación. Los patrones de procesamiento carnicero tienen relación con la procedencia del animal (salvaje o domesticado por el grupo), la forma de caza y su posterior tratamiento hasta la obtención del producto final (alimento, vestimenta, etc.) (Beovide 2007).

El conjunto arqueofaunístico de Puerto La Tuna hace referencia a: $(I)$ animales provenientes de hábitats del humedal, arenal y pradera circundante y complementan la lista de la fauna mencionada en las crónicas, consumidas dentro de los circuitos domésticos (Beovide 2007). (2) Evidencias de descarnado, descuartizamiento y restos con alteración térmica que se recuperan en un contexto con cerámica con residuos de ácidos grasos animales y morteros con pigmentos minerales con posibles residuos grasos (Beovide et al.20 I I, Malán et al.20 I I) lo que sugiere el uso de la fauna en las preparaciones alimenticias, así como en la decoración cerámica y corporal.

Tanto del coipo como del ciervo están representadas partes del esqueleto apendicular en el registro arqueofaunistico del sitio, así como la región de la cabeza del coipo y del carpincho. Se puede sugerir a modo de hipótesis de trabajo (que deberá ajustarse desde distintas vías como la preservación diferencial de restos, entre otras) que los primeros sufrieron un proceso de extracción inicial de la piel para abrigo y fueron consumidas dichas partes (sumado a esto último el carpincho) en el sitio por su importante valor energético o por preferencias alimentarias, entre otras. Los peces y pequeños roedores de los que están representadas distintas partes del esqueleto posiblemente fueron consumidos en el sitio en preparaciones que involucraron la totalidad del animal.
El hervido de la fauna o el asado se propone como mecanismos potencialmente probables de preparación de la misma. Ambos son discutidos a nivel de la preparación de roedores pequeños (Cavia aperea) por Acosta y Pafundi (2005) considerando entre otras cosas la crónica de Paucke (1944) de asado en ceniza. Por otra parte, la termoalteración del conjunto arqueofaunístico también puede obedecer en este caso a la confección de artefactos, a su uso como combustible o ser producto del descarte en los fogones por limpieza o mantenimiento del espacio.

Agradecimientos: Nuestros agradecimientos a: Alejandro Ferrari, Federica Moreno, Elena Vallvé y al equipo del proyecto FCE-ANII-2007-186, así como a los evaluadores del artículo por sus sugerencias.

\section{BIBLIOGRAFÍA}

Acosta, A. y L. Pafundi 2005. "Zooarqueología y tafonomía de Cavia aperea en el humedal del Paraná inferior". Intersecciones en Antropología 6: 59-74.

Behrensmeyer, A. K. 1978. "Taphonomic and ecologic information from bone weathering”. Paleobiology 4 (2): 150-162.

Beovide, L. 2003. "Crónicas del espacio costero, Uruguay". En Anais do XII Congreso da Sociedade de Arqueología Brasileira. Arqueologías da América Latina, editado por J. L. de Morais, M. Coutinho Afonso, D. Candida Martins. CD-ROM, San Pablo.

2007. "Animals, bones and indians: patterns of butchering process in the indigenous economy from 16 th to 18 th centuries a.d. in the Banda Oriental”. En British Archaeological Reports Taphonomy and Zooarchaeology in Argentina, editado por M. Gutierrez, L. Miotti, L., Barrientos, G., Mengoni Goñalons y G. M. Salemme, pp. 150-180. Archaeopress. Oxford, Inglaterra.

201 la. "La presencia de cultígenos desde el quinto milenio en el registro arqueológico del curso medio platense: revisión y perspectivas”. En Avances y Perspectivas en la Arqueología del Nordeste, editado por M. Feuillet, B. Colasurdo, J. Sartori y S. Escudero, pp. 155- 175. Santo Tomé, Argentina.

$201 \mathrm{lb}$ "Las sociedades prehistóricas de la cuenca del río Santa Lucía: una mirada desde la epistemología de la complejidad". En Resúmenes del I Congreso Internacional de Arqueología de la Cuenca del Plata, pp. 4 (en prensa).

Beovide, L., M. Malán; E. Vallvé, A. Trujillo, M. Mejía, H. Pardo, R. Faccio, A. Mombrú, M. Pistón. 20I I. "Contenedores, Instrumentos y Pigmentos: una Aproximación Arqueométrica a los Procesos de Producción y Uso en las Sociedades del Holoceno Tardío en Los Humedales del Santa Lucía”. III Congreso Latinoamericano de Arqueometría, pp. 4-6. Arica, Chile (en prensa).

Binford, L. 1980. “Willow smoke and dogs' tails: huntergatherer settlement systems and archaeological site formation”. American Antiquity 45: 4-20. 
Uso de la Fauna al Momento del Contacto Hispano-Indígena en el Río de La Plata: un Aporte Arqueozoológico y Etnohistórico Press, New York.

198I. Bones:Ancient Men and Modern Myths.Academic

1984. Faunal remains from Klasies River Mouth. Academic Press, Orlando. Bellaterra.

Butzer, K. 1989. Arqueología, una ecología del hombre.

De Nigris, M. 2004. El consumo en grupos cazadores recolectores. Un ejemplo zooarqueológico de Patagonia meridional. Colección Tesis Doctorales, Sociedad Argentina de Antropología, Buenos Aires.

Fernández de Oviedo, G. 185I. Historia General y Natural de las Indias (1535). Real Academia de Historia, Madrid.

García, D. 1933. "La «Memoria» del Diego de García (I526-1527)". Revista de la Sociedad de Amigos de la Arqueología (7): 169-228.

Hedges, R. y A. Millard. 1995."Bones and Groundwater: Towards the Modelling of Diagenetic Processes". Journal of Archaeological Science 22: I55- 164.

Lope de Souza, P. 1958. "Diario de Navegación (15301532)". En Apartado de la Revista de la Sociedad de Amigos de la Arqueología, editado por H.Arredondo, pp. I34-| 140. Editorial Siglo llustrado, Montevideo.
Malán, M., E. Vallvé, A. Malvar y S. Campos. 20I I "Sobre vasijas y sus decoraciones: un acercamiento a sus funciones y usos". En Resúmenes del I Congreso Internacional de Arqueología de la Cuenca del Plata, pp. 161-162.Artículo en prensa.

Mengoni, G. 1988. "Análisis de los materiales faunísticos en los sitios arqueológicos”. Xama I: 7I-I20.

1999. Cazadores de guanacos de la estepa Patagónica. Sociedad Argentina de Antropología, Colección de Tesis Doctorales, Buenos Aires.

Mondini, M. 2002. "Carnivore Taphonomy and the Early Human Occupations in the Andes".Journal of Archaeological Science, 29: 79|-80I.

Paucke, F. 1944. Hacia allá y para acá (una estada entre los indios Mocovies 1749-I767). Instituto Cultural ArgentinoGermano, Universidad Nacional de Tucumán. Instituto Argentino germano, Tucumán.

Payne, S. 1975. "Partial recovery and simple bias". En Archaeozoological Studies, editado por A. Clason, pp. 7-17. NorthHolland, Amsterdam.

Schmidl, U. 1962. "Viaje al Río de la Plata (I534-I554)". En Biblioteca Indiana "Viaje por América del Sur», editado por M. Moliner, (4):276-285. Editorial Aguilar. Madrid. 